\title{
Saint Jean le Russe : pèlerinage et territorialité
}

\section{Katerina Seraïdari}

\section{OpenEdition}

\section{Journals}

Édition électronique

URL : https://journals.openedition.org/geohist/541

DOI : 10.4000/geohist.541

ISSN : 2264-2617

Éditeur

Association française de la Revue de géographie historique

\section{Référence électronique}

Katerina Seraïdari, «Saint Jean le Russe : pèlerinage et territorialité », Revue de géographie historique [En ligne], 16 | 2020, mis en ligne le 20 mai 2020, consulté le 13 juin 2021. URL : http://

journals.openedition.org/geohist/541 ; DOI : https://doi.org/10.4000/geohist.541

Ce document a été généré automatiquement le 13 juin 2021.

\section{(c) $($ () $\ominus$}

Ce(tte) œuvre est mise à disposition selon les termes de la Licence Creative Commons Attribution -

Pas d'Utilisation Commerciale - Pas de Modification 4.0 International. 


\title{
Saint Jean le Russe : pèlerinage et territorialité
}

\author{
Katerina Seraïdari
}

1 Né vers 1690 dans le sud de la Russie, Jean fut soldat dans l'armée de Pierre le Grand. Fait prisonnier, il fut vendu en 1711 à un notable ottoman de Prokopi (Urgup) en Cappadoce. Son maitre voulut le convertir à l'islam, mais le jeune homme résista malgré les tortures infligées. Progressivement, les conditions de vie de Jean s'améliorèrent, même s'il a toujours vécu dans une étable. Sa sainteté aurait été reconnue tant par les musulmans que par les orthodoxes turcophones de la région bien avant sa mort, survenue le 27 mai 1730. Trois ans après son décès, des signes miraculeux conduisirent à son exhumation et sa relique incorruptible, qui révéla des pouvoirs thaumaturges, fut placée dans une église.

2 La biographie de ce saint renvoie à une époque pré-nationaliste qui est caractérisée par des modèles d'identification fluides. D'une part, le lieu de naissance de Jean n'est pas connu et une certaine confusion règne autour de ses origines : Jean pourrait même être né dans un village localisé dans l'Ukraine actuelle. Mais saint Jean reste russe, comme l'adjectif qui suit systématiquement son prénom le montre; il n'est jamais présenté comme Ukrainien. D'autre part, les orthodoxes turcophones de Cappadoce, qui ont établi son culte, sont appelés Karamanlides. Cette population orthodoxe parlait le turc et l'écrivait avec des caractères grecs. À leur égard, les opinions sont partagées, comme Richard Clogg (1999, p.116) le souligne: les historiens grecs affirment qu'ils sont d'origine grecque et ont adopté le turc comme langue soit par obligation (forcés par l'occupant) soit à cause de leur isolement géographique, alors que certains de leurs homologues turcs soutiennent qu'ils sont d'origine turque et se sont installés sur le territoire byzantin bien avant la conquête ottomane ; ils auraient été obligés de suivre la religion de ceux qui gouvernaient l'empire, les Byzantins, mais auraient pu sauvegarder leur langue d'origine. Toutefois, les Grecs ont très tôt considéré les Karamanlides comme leurs compatriotes, alors que les Turcs l'ont fait bien plus tardivement. L'organisation de l'Empire ottoman selon le système de millet (communauté religieuse) et l'existence de pratiques linguistiques très 
mélangées au sein de l'Empire (ibid, p.117-118) expliquent pourquoi les Karamanlides s'auto-définissaient par la religion orthodoxe.

3 Au dix-neuvième siècle, la ville de Prokopi comptait deux églises, dont une en très mauvais état. Le 2 juin 1886, débuta la construction d'une troisième église à l'emplacement de l'ancien cimetière, dans le but de remplacer celle qui menaçait de s'écrouler; dédiée à saint Jean le Russe, elle fut érigée sur sa tombe qui, même vide, restait un important lieu de dévotion (Anonyme, 1938). Il semble qu'à la fin du dixneuvième siècle, l'église de saint Jean le Russe était déjà un important lieu de pèlerinage qui attirait des chrétiens orthodoxes mais aussi des musulmans de toute la Cappadoce, ainsi que de Konya et d'Ankara (évêque Chrysostomos, 2015, p.55). Entre 1850 et 1859 , les habitants hellénophones de la ville voisine de Synasos faisaient des legs au " prisonnier de guerre qui est devenu moine » (ehmaloto ton kalogero) : c'est la période de la guerre de Crimée (1853-1856), et ces legs montrent tant leur dévotion envers Jean que leur sympathie envers la Russie, grande puissance orthodoxe qui pourrait leur venir à aide (Hadziiossif, 2005, p. 58).

Dès le début du vingtième siècle, la montée des nationalismes et le démantèlement de l'Empire ottoman affectèrent ce pèlerinage. Quand les orthodoxes d'Urgup durent partir, après le Traité de Lausanne en 1923, ils emportèrent avec eux la relique du saint, malgré la tentative des musulmans locaux de la garder. Ce sont des critères religieux qui ont prévalu lors de cet échange de populations entre la Grèce et la Turquie - même si l'idée initiale était que les Karamanalindes devaient en être exemptés et autorisés à rester chez eux (Clogg, 1999, p.115).

en Eubée qu'ils appelèrent Neo Prokopi. Selon un de mes interlocuteurs dont le père était venu de Cappadoce, «ce sont les réfugiés qui ont tout créé ici; avant il n'y avait que la montagne, rien d'autre ». L'économie locale fut bouleversée par l'arrivée des réfugiés. Encore aujourd'hui, des commentaires ironiques sont entendus sur les métiers des natifs : «Des apiculteurs qui font du miel, et des résiniers (ritinokalliergites), c'est tout. En revanche, les réfugiés ont toujours eu l'esprit commercial. Ils voyaient loin ».

6 En 1951, une église fut construite en l'honneur de saint Jean. Elle constitue un des lieux de pèlerinage les plus importants du pays qui attire tant des Grecs que des Russes, des Serbes, des Roumains et des Chypriotes. Ce qui la caractérise est qu'elle matérialise la transplantation d'un culte dans un autre pays suite au déplacement d'une population. De ce point de vue, elle rappelle le monastère de la Vierge de Soumela, haut lieu des Grecs pontiques et un des plus grands pèlerinages actuels en Grèce, qui a été transposé de Trébizonde en Macédoine (Bruneau, 1998) : ce n'est pas un hasard si le sanctuaire de saint Jean le Russe fut inauguré le 27 mai 1951, alors que celui de la Vierge de Soumela en août 1952. Cette période, qui marque la fin de la guerre civile en Grèce (1946-1949), est enfin propice pour des initiatives de ce genre.

7 D'un point de vue méthodologique, je m'appuierai sur des données bibliographiques et ethnographiques, recueillies lors d'une première enquête de terrain à Neo Prokopi en octobre $2019^{1}$. Mon objectif est d'analyser l'organisation spatiale tant de l'intérieur de l'église que de son périmètre, ainsi que les multiples dimensions de ce pèlerinage: religieuse, mémorielle et commerciale. Au centre de cet article, se trouve également une autre dimension qui relève de la territorialité : j'entends par là la relation particulière qui est tissée entre un groupe et un territoire donné - définition qui s'inspire de la réflexion de Julien Aldhuy (2008). Aldhuy considère que pour mieux 
appréhender ce que l'espace vécu représente, il faut adopter une perspective diachronique et s'intéresser à la logique d'identification (je suis de là), d'appartenance (c'est chez moi) et d'appropriation (c'est à moi). Toutefois, afin de mieux saisir les réalités de mon terrain, j'utiliserai ici le terme «sentiment de familiarité » au lieu de celui de logique d'appartenance.

8 Comme nous le verrons, certains objets, représentations ou lieux au sein du pèlerinage fonctionnent comme des accroches qui font émerger la dimension de territorialité. Cette dimension fait que l'espace vécu ne renvoie pas seulement à un hic et nunc, mais aussi à un «là-bas » qui est différemment défini selon les personnes - que ce soit la Cappadoce, le Mont Athos ou la Russie.

\section{Organisation de l'espace}

Le pèlerin qui arrive en bus de Halkida, la capitale d'Eubée, descend à l'entrée du village. Autour de lui, des cafés, des magasins, l'école de l'autre côté de la route nationale, mais aucun indice visible de l'église. Pendant la période estivale, s'il arrive après 11 heures, il n'a qu'à suivre l'odeur de viande grillée pour la trouver. En effet, devant les nombreuses tavernes de la localité, sont posés sur le trottoir des petits barbecues sur lesquels des brochettes de viande sont préparées pratiquement toute la journée.

10 Deux noyaux commerciaux se sont développés dans le village montagnard de Neo Prokopi. Si le premier est défini par sa proximité avec la route principale, le second l'est par rapport au sanctuaire, qui se trouve aux confins du village. Les photographies de l'époque de la construction de l'église montrent qu'il n'y avait tout autour que des arbres, des champs et quelques cabanes pour les outils ou les animaux. C'est donc après cette date que le second noyau commercial commença à émerger. Par exemple, le cafépâtisserie, tout près de l'église, fut créé en 1958, comme une plaque sur la façade le rappelle. Le développement de ces commerces est associé à l'affluence des pèlerins qui n'a fait que grandir au fil des années.

11 Ce second noyau commercial est composé de lieux de restauration et de détente pour les pèlerins, mais aussi de stands de vente d'objets religieux et de spécialités locales, comme le miel (photo 1). Il se situe dans le prolongement du premier, d'où l'impression qu'il suffit de suivre l'odeur de viande grillée pour découvrir l'église. Le cadre spatial de Neo Prokopi est donc défini par un dédoublement : les magasins autour du sanctuaire s'adressent aux pèlerins qui affluent entre avril et octobre; en revanche, les commerces qui sont plus bas, au bord de la nationale, sont fréquentés par une clientèle locale, été comme hiver. Si un grand nombre de places de stationnement est réservé aux pèlerins qui sont motorisés ou voyagent en cars (dans le cas des excursions paroissiales), les magasins d'en bas ne disposent pas d'un tel aménagement. Cette configuration spatiale rappelle, dans une certaine mesure, la dualité de la ville de Lourdes (Chadefaud, 1981).

12 Les commerçants autour du sanctuaire sont, dans leur grande majorité, des descendants des réfugiés, alors que ceux d'en bas sont principalement des natifs (dopioi). Toutefois, si la différence entre réfugiés et natifs était significative auparavant, elle l'est beaucoup moins aujourd'hui, à cause des mariages mixtes et de la mobilité croissante des personnes : certains descendants des réfugiés ont même immigré aux États-Unis. Selon Vasso Stelaku $(2008$, p.187) qui a fait ses enquêtes ethnographiques à 
Neo Prokopi entre 1996 et 1997, les réfugiés qui avaient des propriétés dans cette partie du village étaient fiers des liens de voisinage qu'ils entretenaient avec le saint.

Ce noyau commercial fonctionne de manière autonome par rapport au reste du village. En effet, de nombreux pèlerins ne sortent pas de cet espace qui est spécialement conçu pour satisfaire leurs besoins et où les commerces proposent des menus rapides pour ceux qui sont en cars et doivent partir à des horaires précis; ou des menus spéciaux selon la nationalité des visiteurs. Comme la serveuse d'un café près de l'église me l'a expliqué, les pèlerins russes ne vont jamais dans les cafés ; dès le matin, ils veulent manger, c'est pour cette raison qu'une taverne voisine prépare spécialement pour eux des œufs et des saucisses pour le petit-déjeuner.

Mais ce noyau n'est pas seulement défini par des fonctions cultuelles ou commerciales : on y trouve également un musée et un bâtiment où les pèlerins peuvent être logés. En Grèce, il est courant d'avoir des aménagements de ce genre près d'un lieu de pèlerinage, surtout quand celui-ci est excentré. Michel Bruneau (1998, p.225) se réfère aux chambres d'hôtes (xenonas) pour les pèlerins, qui ont été construites par différentes associations pontiques dès 1963, près du monastère de la Vierge de Soumela. Dans tous ces cas, le nom « hôtel » (xenodoheio) n'est jamais utilisé pour ne pas associer cette offre de logement à une intention commerciale.

Document 1 : Stand à proximité de l'église avec des icônes de saint Jean le Russe et d'autres saints, où sont également vendus des produits locaux (figues séchées, pistaches, miel). En arrièreplan, on distingue une taverne et sous le parasol, un homme debout qui s'occupe de la viande sur le barbecue.

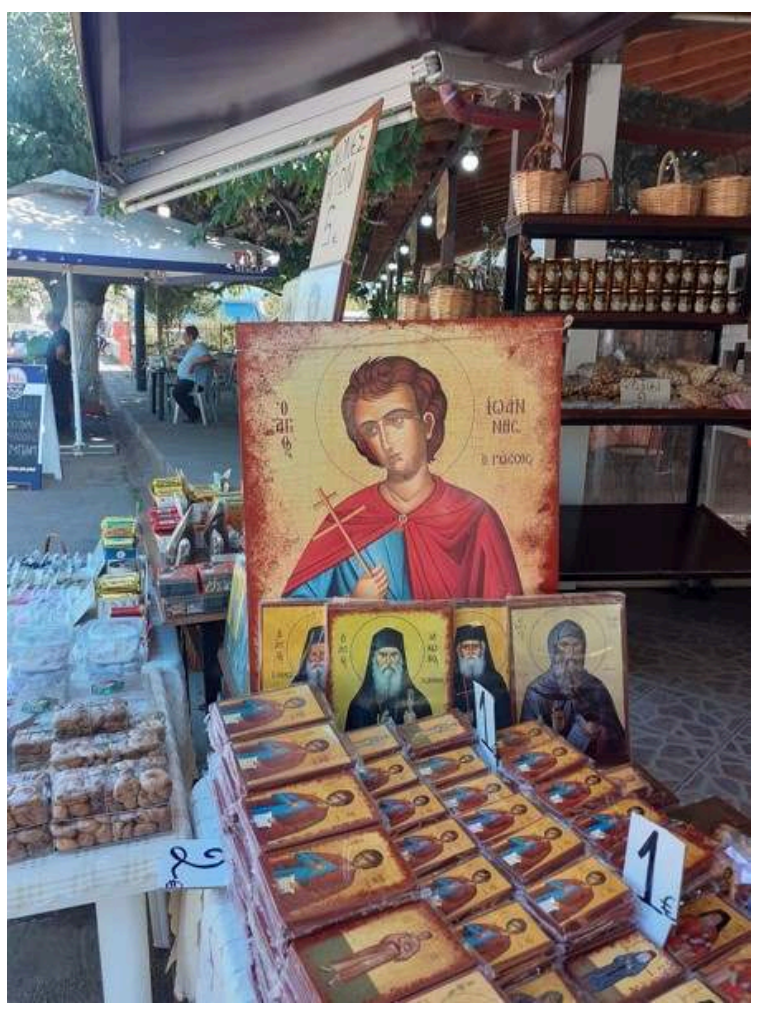

Source : Katerina Seraïdari 


\section{A. Chambres d'hôtes et Musée} 1989, comme une plaque commémorative le précise ; une représentation du Patriarcat de Moscou était présente à cette occasion. Il a une capacité d'accueil de 160 lits et la plupart des chambres ont vue sur l'église, selon le site Internet du pèlerinage ${ }^{2}$. Dans la réception, on trouve des objets évoquant la Cappadoce, des photographies d'ecclésiastiques ou de personnes qui ont joué un rôle important dans le transfert de la relique en Grèce et l'établissement du pèlerinage, ainsi qu'un tableau, fait en 2002, qui représente une cathédrale russe, celle de Saint-Sauveur de Moscou. Selon le responsable du xenonas, c'est pour que les pèlerins russes qui viennent massivement rendre hommage au saint se sentent " chez eux » pendant leur séjour. Ce tableau n'est pas seulement un signe de bienvenue. Il fonctionne aussi comme une accroche qui puise son efficacité dans l'évocation d'un lieu emblématique; un simple élément décoratif, qui sert à rendre familier un lieu où les pèlerins russes n'ont pas d'attaches, peut ainsi être investi d'une dimension de territorialité. En somme, ce qui est en jeu ici est le sentiment de familiarité. Civilisation d'Asie Mineure (Mouseio Mikrasiatikou Politismou). Inauguré le 10 septembre 2018, il abrite un certain nombre d'objets que les réfugiés ont apportés de Cappadoce : des icônes, des objets ecclésiastiques mais aussi de la vie courante, des livres, des photos, des costumes, des broderies, ainsi que des bijoux que les dévots ont déposés, au fil des années, auprès du saint comme offrandes. Les pancartes du musée sont en trois langues, en grec, en anglais et en russe - ce qui montre, encore une fois, la priorité que les responsables du pèlerinage accordent au développement du tourisme russe (Seraïdari, 2013 et 2019). Parmi les icônes qui sont exposées, certaines portent des inscriptions en karamanlidika, c'est-à-dire en turc écrit avec des caractères grecs écriture qui n'est compréhensible qu'à ceux qui parlent le turc et savent lire l'alphabet grec. Même si, selon mes différentes sources, des musulmans de Cappadoce viennent encore rendre visite au saint en Eubée, ceux-ci parlent uniquement le turc et sont incapables de déchiffrer l'écriture grecque. Ces inscriptions en karamanlidika sont donc illisibles tant pour les habitants de Neo Prokopi (natifs ou descendants des réfugiés) que pour les pèlerins. ${ }^{3}$

17 Les objets qui y sont exposés proviennent majoritairement d'Asie Mineure (terme utilisé de manière générique), mais aussi de Constantinople, de Cappadoce et quelques uns de "Prokopi de Cappadoce ". Parmi les textiles, certains viennent de Grèce et même d'Eubée; un grand nombre de bijoux du dix-neuvième et du vingtième siècle viennent également d'Eubée ou d'autres régions grecques. Dans ce cas, il s'agit très probablement d'offrandes au saint, après que la relique ait été transférée en Grèce.

Il existe pourtant des artefacts qui ont une provenance étrangère et pour lesquels il est attesté qu'ils ont été offerts au saint bien avant son transfert. Il s'agit de quatre objets ecclésiastiques, fabriqués en Russie au dix-neuvième siècle : deux patènes (où le pain est placé lors de la cérémonie eucharistique) qui représentent le Golgotha, une en bronze plaqué or (sans date de fabrication précise), et une autre en argent, fabriquée en 1869 ; un calice en argent plaqué or de 1874 ; et, enfin, une custode (pour transporter le pain béni) en argent plaqué or, datant de 1860 . 

des liens entre le saint et le peuple russe; ils peuvent ainsi appuyer un certain sentiment de familiarité de la part des pèlerins russes. En effet, ces derniers ne sont pas des étrangers dans ce lieu, non seulement parce que le saint qui y est vénéré était un compatriote (la possibilité d'une origine ukrainienne n'étant jamais évoquée dans les pancartes au sein du pèlerinage), mais aussi grâce à ces objets qui les inscrivent dans une lignée continue de pèlerins russes. C'est leur capacité de donner de la profondeur historique au sentiment de familiarité (ce que le tableau représentant Saint-Sauveur de Moscou ne peut pas faire, par exemple) qui transforme ces artefacts en marqueurs de territorialité.

\section{B. Le Mont Athos et les Russes}

La vitrine avec les objets ecclésiastiques russes se trouve en face d'un ancien reliquaire du saint ; il est en bois de cyprès et représente saint Jean les yeux fermés et les mains croisées sur sa poitrine, comme un gisant. Les visiteurs ne peuvent pas voir l'intérieur $\mathrm{du}$ reliquaire qu'il suffirait pourtant d'ouvrir pour constater que le saint est, sur ce côté, représenté avec les yeux ouverts et tenant une croix dans la main droite. Cette double représentation véhicule une idée largement répandue parmi ceux qui vénèrent la relique : il ne faut pas se fier aux premières impressions et considérer le saint comme mort ; les apparences trompent, car le saint est un être vivant.

21 Selon la pancarte du musée, ce reliquaire a abrité le corps du saint de 1860 jusqu'aux «premières années de l'arrivée dans sa nouvelle patrie, Prokopi d'Eubée ». La pancarte précise que c'est une offrande du « monastère de Panteleimon du Mont Athos à l'église du saint à Prokopi de Cappadoce qui venait d'être construite (ston neoktisto nao)». Les informations de la pancarte sont contradictoires, dans la mesure où l'église du saint en Cappadoce, dont la construction commença en 1886, ne fut inaugurée qu'en 1892 - l'un des deux clochers étant encore aux travaux en 1910 (évêque Chrysostomos, 2015 : 140-141), alors que le reliquaire est censé être utilisé depuis 1860. Quoi qu'il en soit, comme mes informateurs me l'ont affirmé, ce reliquaire constitue aussi une offrande des Russes envers le saint avant l'exode vers la Grèce.

Le monastère de Panteleimon au Mont Athos rassemble, au moins depuis la fin du dixneuvième siècle, une nette majorité de moines russes. Les érudits grecs de l'époque ont beaucoup écrit sur l'évolution de la composition du monastère. Sotirios Koutroubas (1900, p.29-30), par exemple, considère que des Russes motivés par des intentions expansionnistes (kataktitikon skopon) arrivèrent au Mont Athos en 1830 et que sous leur action le monastère devint russe en 1875. Ioannis Tantalidis (1874, p.53), qui est plus modéré, insiste sur le caractère changeant de ce monastère au fil des siècles : il fut slavo-russe, serbe, purement russe, purement grec avant de devenir dans les années 1870 «russo-grec», ayant 300 moines russes et 200 grecs. Les quatre objets ecclésiastiques russes mais aussi le reliquaire renvoient donc à une période pendant laquelle les Russes sont accusés de vouloir s'approprier le Mont Athos qui fait encore partie de l'Empire ottoman, et suscitent la méfiance en Grèce. En effet, la fin de la guerre de Crimée en 1856 marque la montée graduelle d'un sentiment antirusse en Grèce (Skopetea, 1988, p.331). Mais rien de tout cela ne transparaît dans l'organisation du Musée de Civilisation d'Asie Mineure. 

initialement placé à l'entrée de l'église de Neo Prokopi afin que les pèlerins puissent lui rendre hommage ; il est aussi présenté comme un « cadeau du monastère russe de saint Panteleimon» (évêque Chrysostomos, 2015, p.64, note 118). Ce qui signifie qu'abstraction est faite, ici également, de tous les débats qui ont agité une partie des érudits et des politiques grecs de la fin du dix-neuvième siècle pour ne garder qu'une information neutre car simplifiée : ce monastère est actuellement russe et l'était aussi au moment de la fabrication du reliquaire ${ }^{4}$.

Les objets russes, que les réfugiés transportèrent avec eux-ce qui montre toute l'importance qu'ils leur accordaient - témoignent du rayonnement de ce lieu de culte cappadocien : même les Russes vénéraient saint Jean. Une fois dissociées de tout contexte historique, les offrandes russes ne sont qu'une source de prestige, la preuve matérielle que le culte du saint $a$, très vite, dépassé le cadre local pour arriver jusqu'au Mont Athos et même en Russie. Grâce à ces cadeaux mais aussi à l'aide financière russe pour la construction de l'église en Cappadoce (dont les clochers étaient en forme d'oignon - caractéristique architecturale typiquement russe), le pèlerinage peut se vanter de son caractère international dès le dix-neuvième siècle.

La dimension de territorialité dont ces objets sont investis varie forcément selon les personnes : s'ils servent d'appui pour le développement d'un sentiment de familiarité en ce qui concerne les pèlerins russes, dans le cas des descendants des réfugiés mais aussi de tout visiteur grec, nous sommes plutôt dans une logique d'appropriation (« un saint si important, et pourtant il a toujours voulu rester avec nous!»).

\section{Pratiques de pèlerinage}

Les chambres d'hôtes, le musée et l'église constituent un ensemble cohérent, non seulement à cause de leur emplacement contigu mais aussi parce qu'ils sont tous dirigés par les responsables du pèlerinage. Toutefois, beaucoup de pèlerins, surtout grecs, viennent simplement rendre hommage au saint sans visiter le musée ou dormir au xenonas. Pendant mes enquêtes de terrain, j'ai rencontré une femme de Halkida qui est venue en bus le matin afin de passer quelques heures dans l'église avant de repartir chez elle en début d'après-midi ; deux cyclistes qui se sont arrêtés pour prier auprès du saint et boire un rafraichissement dans un café avant de continuer leur tour de vélo dans les montagnes entourant Neo Prokopi; et, enfin, un couple d'Athéniens d'une cinquantaine d'années qui est venu d'Athènes jusqu'à Halkida en bus (trajet d'environ une heure) et qui a ensuite pris un bus local pour Neo Prokopi (trajet d'une heure également) : après une courte visite au saint, ils sont retournés chez eux le soir de la même manière. La notion de pèlerinage peut donc renvoyer tant à une visite, plus ou moins, rapide de pèlerins isolés (qui habitent, le plus souvent, en Eubée, à Athènes ou dans d'autres régions voisines) qu'à un séjour prolongé. Si la première forme correspond à une décision spontanée qui ne nécessite pas une grande organisation, la seconde nous fait entrer dans la logique du pèlerinage à dimension collective, qu'il soit organisé par une paroisse, une autre instance religieuse ou par une agence de tourisme (Seraïdari, 2019).

27 Bien que le nombre de visiteurs soit difficile à estimer, le prêtre qui était responsable de l'église en 2009 considérait que celle-ci recevait en été entre douze et quinze mille personnes par semaine (Vernezos, 2009, p.2). Si le transport ferroviaire a joué un rôle

Revue de géographie historique, 16 | 2020 
considérable dans l'émergence de Lourdes comme pôle d'attraction majeur (Chadefaud, 1981), le développement du pèlerinage à destination de saint Jean le Russe a été facilité, d'une part, par la proximité avec Athènes ; et d'autre part, par l'existence d'un réseau de transport en commun assez efficace, qui est densifié pendant l'été mais aussi pendant la fête du saint (26-27 mai). Même les pèlerins étrangers les plus fortunés peuvent opter pour une brève visite - même si cela exige le déploiement de moyens importants: mes interlocuteurs ont mentionné l'arrivée spectaculaire d'une riche femme russe, qui est venue en hélicoptère depuis Athènes ; étant donné que le village ne dispose pas d'héliport, elle a atterri sur le stade de football, où un taxi l'attendait pour l'amener directement à l'église.

Saint Jean le Russe est fêté deux fois dans l'année, le 27 mai (commémoration de son trépas) et le dernier dimanche du mois de septembre (commémoration de l'inauguration de son église en Eubée, qui a eu lieu le dimanche 21 septembre 1969). Bien évidemment, seule la première fête était célébrée en Cappadoce. Le culte de ce saint a donc connu une relocalisation. Autre lieu, autres pratiques: d'un côté, une nouvelle fête fut ajoutée en 1970 ; et de l'autre, depuis quelques années, des centaines de pèlerins font à pied les cinquante kilomètres qui séparent Neo Prokopi de Halkida, la capitale d'Eubée, afin d'assister aux festivités de mai. Cette pratique pénitentielle est censée être inspirée d'une guérison miraculeuse accordée à un enfant : son père l'avait apporté à Neo Prokopi à pied, sur son dos, après un trajet de deux jours et demi.

Écourtées ou prolongées, cherchant la facilité ou multipliant les difficultés qu'il faut surmonter, les pratiques qui se développent au sein de ce pèlerinage restent variables. Une variabilité similaire caractérise la manière dont les dévots se comportent à l'intérieur de l'église.

\section{A. L'organisation de l'espace à l'intérieur du sanctuaire}

Après avoir allumé une bougie à l'entrée de l'église (où se trouvent également des livres et d'autres objets religieux en vente), le pèlerin entre dans la partie centrale, où la première chose à faire est de rendre hommage à la relique. L'itinéraire du visiteur est balisé, sur sa gauche, par une barrière en marbre. À sa droite, le reliquaire en argent est posé sous la réplique d'un caveau en marbre. La rencontre avec le saint est complétée par l'hommage rendu à son icône, qui est couverte de bijoux et d'ex-voto et qui représente le saint tenant une croix dans la main droite et un parchemin dans la main gauche (photo 2). 
Document 2 : La barrière en marbre qui balise l'itinéraire que les visiteurs doivent suivre à l'intérieur de l'église.

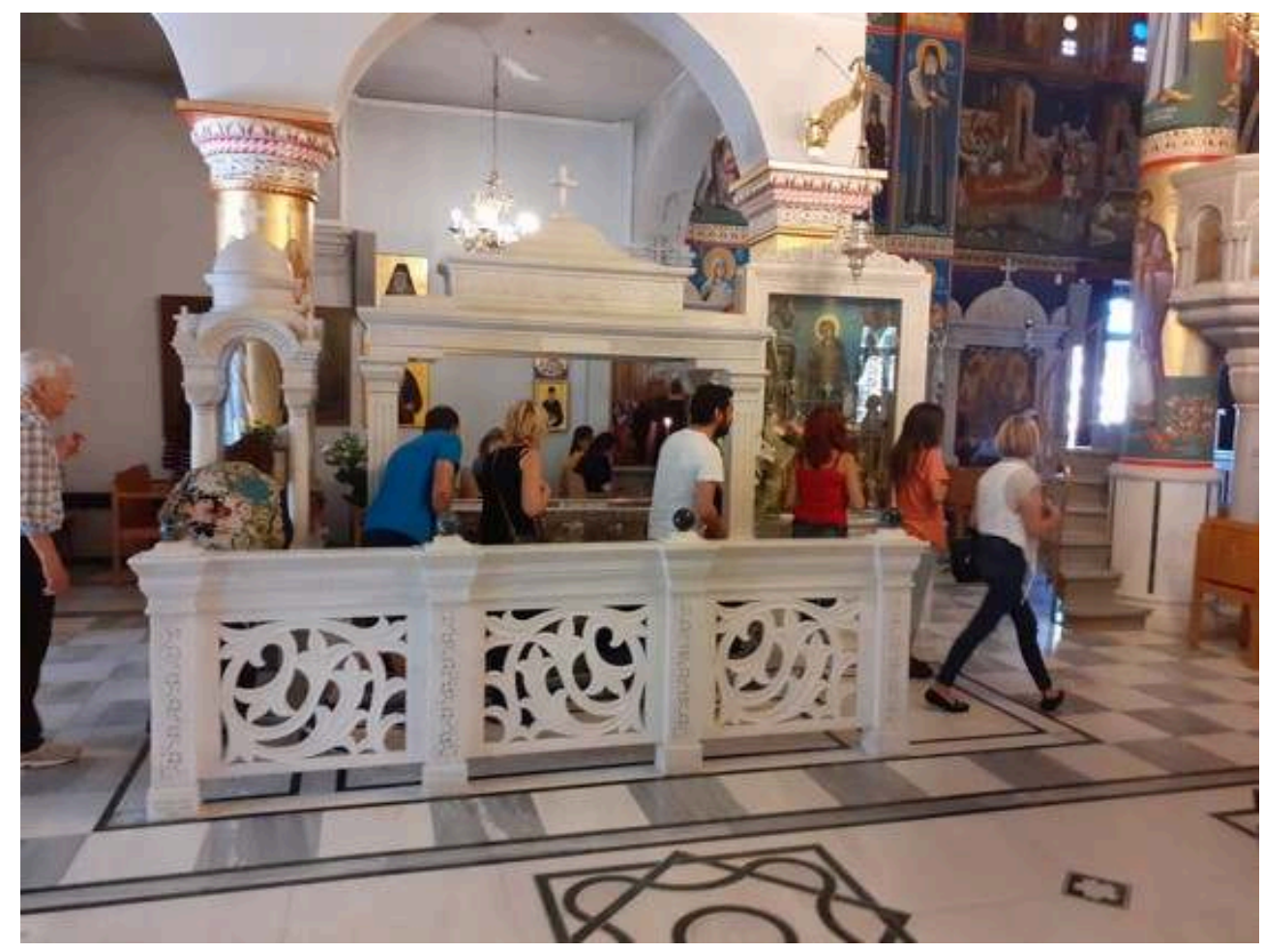

Source : Katerina Seraïdari

Après avoir rendu hommage à la relique, le visiteur peut se promener dans l'église afin de regarder les fresques qui racontent l'histoire de son transfert de Cappadoce en Eubée; ou saluer les icônes de l'iconostase, dont certaines ont été peintes au Mont Athos à la fin du dix-neuvième siècle par l'iconographe Daniil Katounakiotis pour l'église de Cappadoce, et transférées en Grèce après l'exode. Il peut aussi sortir de l'église pour visiter les deux chapelles latérales : dans la première (à l'entrée de laquelle des barrières sont posées pour réguler la circulation), les dévots peuvent porter une réplique de la ceinture et du bonnet (skoufaki) du saint - textiles censés avoir des vertus thérapeutiques ; dans la seconde, ils remplissent des bouteilles d'eau et d'huile qu'ils apporteront chez eux ou offriront à des proches (photo 3). Enfin, il peut passer de l'autre côté du reliquaire : assis sur une chaise ou appuyé contre le reliquaire, il peut tranquillement prier et dialoguer avec le saint. Ceux qui veulent déposer des fleurs, offrir un cadeau au saint, ou noter et donner les noms de proches pour la célébration d'une commémoration liturgique préfèrent venir de ce côté où aucune barrière n'est placée. 
Document 3 : La seconde chapelle latérale, où les pèlerins remplissent des bouteilles d'huile et d'eau. L'inscription sur le mur, qui indique que c'est un lieu de bénédiction, est en grec et en russe.

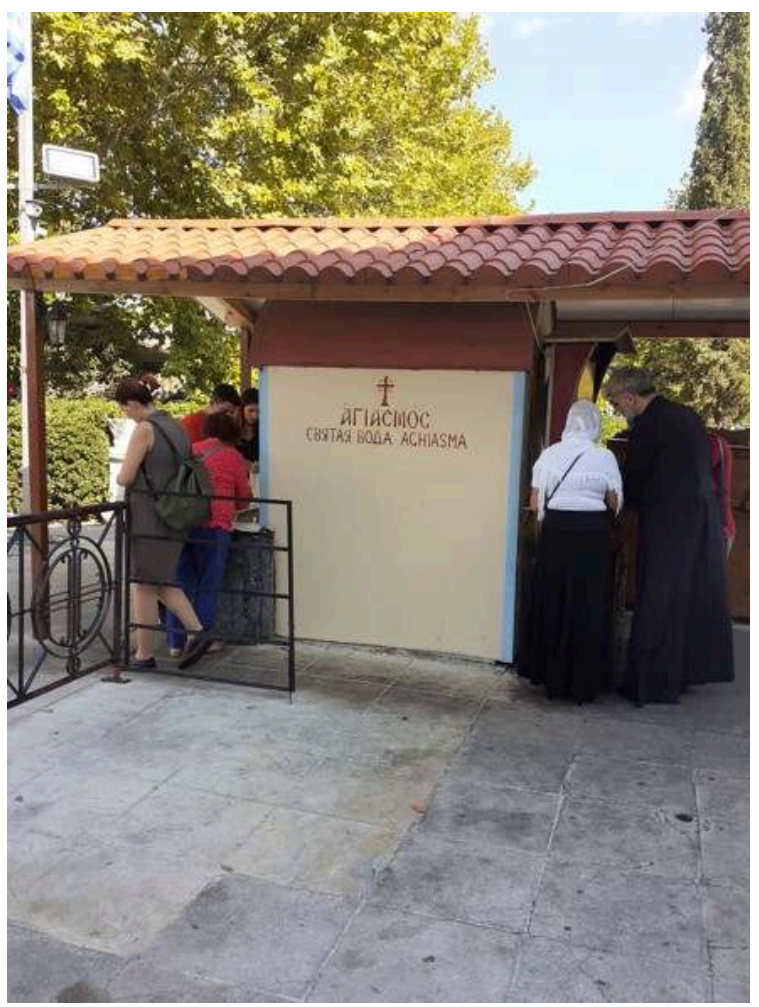

Source : Katerina Seraïdari

Chaque côté du reliquaire est donc soumis à une fonction différente : au côté « public » qui correspond à un passage obligé, se juxtapose le côté «intime» où les dévots peuvent s'attarder et même faire une bénédiction. C'est ce qui se passe souvent avec les pèlerins russes qui viennent en groupe: le prêtre qui les accompagne fait une bénédiction en russe à cet endroit ; les femmes peuvent ainsi s'agenouiller, en utilisant les coussins détachables des chaises afin d'être dans une position plus confortable. C'est dans cet espace « intime » que les pèlerins sont le plus actifs. Il faut pourtant savoir que ce n'est pas seulement l'existence de la barrière qui distingue les deux côtés du reliquaire ; la position du corps du saint le fait aussi, puisque sa tête est inclinée vers le côté " public » : ceux qui restent du côté « intime » et s'adressent à lui ont forcément l'impression que le saint regarde ailleurs, qu'il a le visage détourné.

Paradoxalement, le saint qui est peint sur la colonne en face du côté «intime » est inconnu des pèlerins grecs. Il s'agit d'un saint russe, saint Serge de Radonège (1314-1392), qui a fondé le monastère de la Trinité près de Moscou (photo 4). Ce choix reflète la volonté des responsables du pèlerinage de créer une sorte de « coin russe » initiative qui vise, comme les précédentes, à renforcer le sentiment de familiarité chez les pèlerins russes. 
Document 4 : La colonne avec saint Serge de Radonège. Une employée passe le balai devant le reliquaire, à l'intérieur duquel on distingue la tête du saint. La canne qui est accrochée au caveau de marbre évoque la guérison miraculeuse d'une femme âgée chypriote le 11 août 1978.

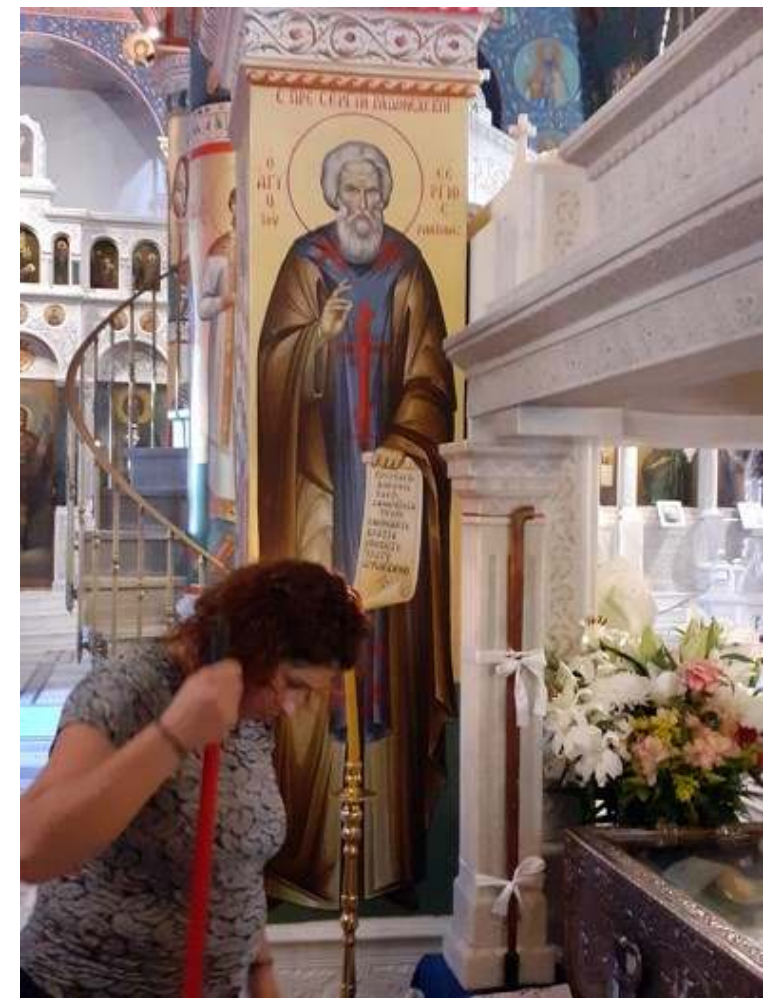

Source : Katerina Seraïdari

\section{B. L'empreinte russe}

La fresque de saint Serge de Radonège porte des inscriptions en grec et surtout en russe. Comme l'un des responsables du sanctuaire me l'a expliqué, les traducteurs russes avec qui ils collaborent pour organiser les visites des pèlerins russes, les ont aidés pour les traductions. «Ils nous ont donné un saint », continue-t-il, suggérant ainsi que ce coin constitue une sorte de contre-don, avant d'ajouter que plus de trois mille Moscovites viennent chaque année pour rendre hommage à saint Jean en Eubée auxquels il faut ajouter les Russes du reste du pays.

À côté de saint Serge, sur la même colonne, le visiteur peut découvrir deux autres saints, bien plus récents : un autre saint russe, Jean de Cronstadt (1829-1908), mais aussi un saint pontique, Georgios Karslidis (1901-1959), qui s'installa en 1930 en Grèce du nord, dans la région de Drama, où il fonda un monastère en 1938 ; toutefois, il était né en Géorgie, d'où ce choix de le placer entre deux saints typiquement russes (photo 5). 
Document 5 : Les deux autres saints de la colonne qui forme le coin russe, saint Jean de Cronstadt et, de l'autre côté, saint Georgios Karslidis. Une femme blonde est agenouillée devant le reliquaire, alors qu'un prêtre russe fait une bénédiction.

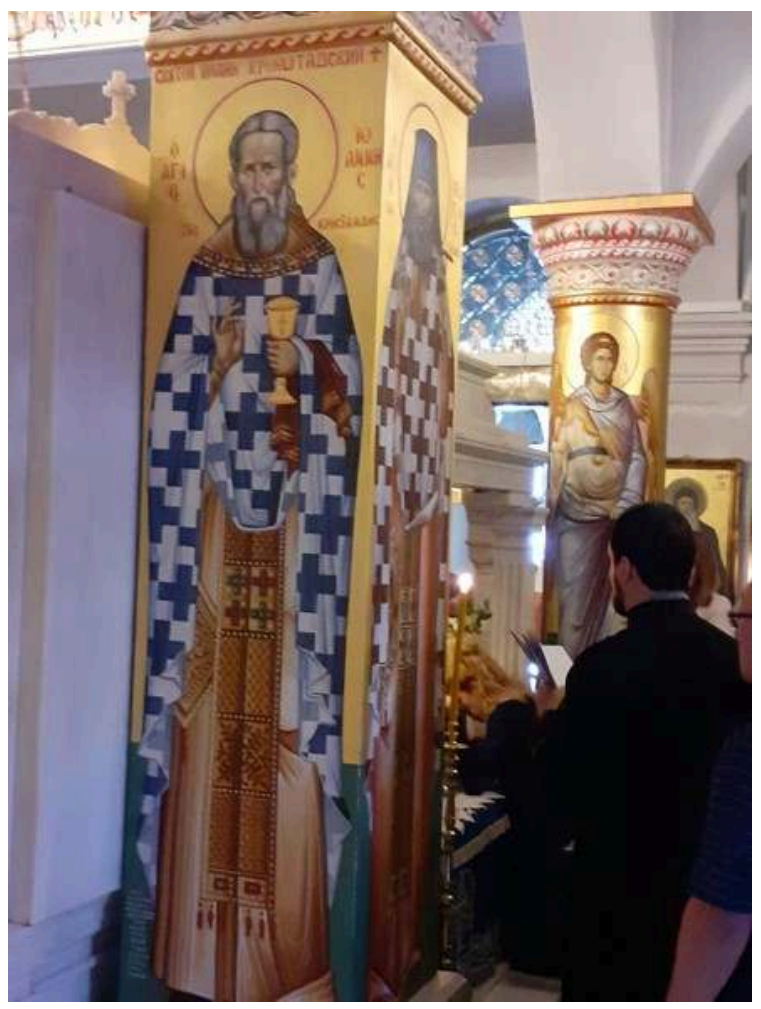

Source : Katerina Seraïdari

Un certain nombre de fresques de l'église ont été financées et commandées par des dévots russes. Selon le même interlocuteur, les Russes savent qu'ils ne peuvent pas avoir le corps du saint mais veulent, tout de même, « laisser leur empreinte » (n'afisoun tin sfragida tous). En effet, il suffit de lire attentivement le livre de l'évêque Chrysostomos pour constater que cette stratégie du compromis est pratiquée depuis la fin du dix-neuvième siècle, quand la relique était encore en Cappadoce : en 1880, les moines russes de saint Panteleimon au Mont Athos envoyèrent un des leurs à Prokopi pour demander une partie de la relique ; après un refus initial et le retour du moine les mains vides, les orthodoxes de Prokopi changèrent d'avis «comme par miracle » et spontanément, décidèrent de couper l'avant-bras droit du saint et de l'offrir au monastère athonite. La délégation arriva au Mont Athos en mai 1881. À leur tour, les moines offrirent des objets ecclésiastiques (iera anathimata) et une somme d'argent pour construire une petite chapelle sur la tombe du saint (évêque Chrysostomos, 2015, p.61).

Les premières pierres de l'église du saint en Cappadoce furent posées en 1886 : c'est donc avec l'argent offert par les moines russes, une fois le bras droit du saint amené au Mont Athos, que la toute première église en son honneur put être érigée. Haut lieu de l'Orthodoxie, le Mont Athos devient ici l'intermédiaire entre le saint et sa patrie, la Russie : «Il semble que le saint a consenti avec empressement à ce que son bras droit soit coupé et envoyé dans ce monastère, pour que par son biais, comme s'il était un clairon tonitruant, la réputation de sa sainteté puisse être diffusé dans toute son immense patrie, la Russie» (Anonyme, 1938, p.22-23). En fin de compte, la description 
$\mathrm{du}$ « coin russe » ne nous permet pas seulement de comprendre la composition d'un décor et les raisons qui ont conduit à ce choix spécifique, mais aussi de prendre en compte le rôle fondamental que les Russes ont joué dès le début dans l'établissement de ce culte. Cette partie près du reliquaire et du saint (dont le bras métallique constitue un rappel du partage) spatialise ainsi la relation "donnant-donnant» qui unit les coreligionnaires russes et grecs.

\section{Modèles d'identification, familiarité et appropriation}

$38 \mathrm{Si}$, depuis des décennies, l'origine des Karamanlides constitue un objet de dispute entre Grecs et Turcs, la question ne se pose pas pour les descendants des réfugiés de Neo Prokopi : leurs ancêtres étaient grecs et, selon la légende, les Turcs n'auraient pas hésité à couper la langue de tous ceux qui persistaient à parler grec afin de réussir à leur imposer le turc (évêque Chrysostomos, 2015, p.163). La turcophonie serait donc le résultat de violences exercées sur eux. De ce point de vue, les icônes qui ont des inscriptions en karamanlidika et qui se trouvent actuellement tant dans le musée que dans l'église, renvoient à une culture à jamais disparue mais qui est méticuleusement sauvegardée par un devoir de mémoire. Ces icônes fonctionnent comme des accroches qui font émerger une dimension de territorialité particulière : de la nostalgie pour ces terres perdues (où saint Jean vécut également pendant vingt ans), mais aussi un sentiment de malaise par rapport à une turcophonie mal assumée car mal connotée.

L'exode a renforcé la relation des orthodoxes de Cappadoce avec saint Jean qui leur offrait un modèle auquel ils pouvaient facilement s'identifier : celui de l'étranger qui finit par être pleinement adopté par une communauté locale; d'ailleurs, celui qui est aujourd'hui considéré comme un des saints les plus populaires en Grèce, ne parlait pas non plus le grec, puisque tant les chrétiens que les musulmans parlaient le turc à Prokopi. Les différentes dimensions de territorialité que ce pèlerinage fait émerger ne sont donc pas seulement liées à des objets et des fresques, le saint lui-même se trouvant au centre de négociations territoriales. Parlant probablement le turc comme tous ceux qui habitaient à Prokopi, le saint a obstinément refusé de se convertir à l'islam, exactement comme les orthodoxes de Prokopi qui-leurs descendants aiment le répéter - ont préféré sacrifier et perdre l'usage de la langue grecque plutôt qu'abdiquer la foi orthodoxe. Après l'échange de populations, le saint a partagé leur destin de réfugié. La dimension de territorialité vient ainsi soutenir la chaîne d'identifications qui font du saint le miroir de ceux qui ont construit et diffusé son culte.

Si le passé des Karamanlides que certaines icônes évoquent, renvoie à un modèle d'identification qui pose problème à cause de la turcophonie, dans le cas des pèlerins russes, les marqueurs de territorialité sont soumis à une fonction différente : tracer les limites entre familiarité et appropriation. En effet, le développement de ce pèlerinage constitue la preuve que le saint, qui est considéré comme un être vivant, a choisi de rester en Eubée, même s'il est d'origine russe et que son bras droit se trouve dans le monastère russe $\mathrm{du}$ Mont Athos. Toutefois, tout est mis en œuvre pour que les compatriotes du saint, les Russes, se sentent chez eux dans ce lieu : d'où les objets et les représentations que ceux-ci reconnaissent comme faisant partie de leur propre culture. En effet, les saints russes peints sur les colonnes de l'église ou le tableau représentant un sanctuaire russe emblématique dans le xenonas n'attirent l'attention que des 
pèlerins russes ; pour tous les autres visiteurs, ils ne sont ni facilement identifiables ni particulièrement significatifs.

41 Il en va différemment des offrandes russes du dix-neuvième siècle, qui sont exposées dans le musée. Alors que pour les pèlerins russes, ces artefacts donnent de la profondeur temporelle à la relation qu'ils entretiennent avec le saint, pour les descendants des réfugiés, ils sont une source de fierté (en tant que preuve de la réputation internationale de leur saint) mais aussi une forme d'héritage légué par leurs ancêtres cappadociens. Mieux encore : ils peuvent être assimilés à des signes révélant l'élaboration d'une conscience nationale grecque, puisque pendant cette période d'affrontements militaires entre l'Empire russe et l'Empire ottoman (avec la guerre de Crimée d'abord, puis avec la guerre russo-turque de 1877-1878), les orthodoxes de l'Anatolie, hellénophones ou turcophones, soutiennent massivement le premier.

Les échanges entre les orthodoxes turcophones de Prokopi et les Russes, qui se formalisent dès 1881 (date de la déposition du bras droit du saint au monastère de Panteleimon), peuvent donc être considérés comme une preuve d'allégeance: les turcophones de Prokopi continuaient de soutenir les Russes, même après leur défaite militaire de 1878. C'est pour cette raison que les hommes d'Église qui écrivent sur saint Jean évitent la question épineuse de la « russification » du Mont Athos : les Russes sont généralement perçus comme des coreligionnaires et des alliés, et ces qualifications étaient encore plus significatives pour les orthodoxes de l'Empire ottoman à la fin du dix-neuvième siècle, vu le rôle de protecteur dont la Russie s'était investie. En fin de compte, si la turcophonie et les karamanlidika introduisent un certain désordre dans les schémas de la rhétorique nationale grecque, ce désordre peut redevenir ordonnable grâce aux contacts avec les moines grecs du Mont Athos (dans le cas des commandes passées à l'iconographe Daniil Katounakiotis, par exemple) mais aussi avec les Russes. De manière paradoxale, la référence aux Russes transforme la discontinuité en continuité, puisqu'elle parvient à « dés-ottomaniser » les turcophones de Prokopi.

L'analyse de la dimension de territorialité fait émerger - derrière le hic et nunc du pèlerinage - des représentations, des négociations, ainsi que d'autres espaces (comme la Cappadoce, le Mont Athos ou la Russie) dont l'interconnexion dépend des pérégrinations de saint Jean pendant sa vie mais aussi post mortem.

\section{BIBLIOGRAPHIE}

Aldhuy J., 2008, « Au-delà du territoire, la territorialité ? », Géodoc, n.55, p.35-32.

Anonyme, 1938, Vie et liturgie de notre père, saint Jean le Russe, le Confesseur de Prokopio de Cappadoce, dont la relique apportée dans son intégrité par les réfugiés de Prokopio est gardée à Neo Prokopio (AhmetAgha) d'Eubée, Athènes, Typois : N. Tilperoglou, 44p. (en grec)

Bruneau M., 1998, «Les monastères pontiques en Macédoine. Marqueurs territoriaux de la diaspora ", dans Bruneau M. (dir .), Les Grecs pontiques. Diaspora, identité, territoires, Paris, CNRS Éditions, p.213-228. 
Clogg R., 1999, « A millet within a millet : the Karamanlides », dans Gondicas D., Issawi C. (dir), Ottoman Greeks in the Age of Nationalism. Politics, Economy, and Society in the Nineteenth Century, Princeton, The Darwin Press, Inc., p.115-142.

Chadefaud M., 1981, Lourdes : un pèlerinage, une ville, Aix-en Provence, Edisud, 123p.

Evêque Chrysostomos, 2015, Saint Jean le Russe. Le " polyathlos » et le champion de patience, Athènes, Apostoliki Diakonia tis Ekklisias tis Ellados, 280p. (en grec).

Hadziiossif Ch., 2005, Synasos. L'histoire d'un endroit sans histoire, Irakleio, Presses Universitaires de Crète, 2005, 479p. (en grec).

Koutroubas S. Ch., 1900, Le Mont Athos d'un point de vue national, Athènes, 115p. (en grec).

Seraïdari K., 2013, « Pèlerinages entre la Grèce et la Russie : jeux de pouvoirs et recompositions du christianisme orthodoxe ", Slavica Occitania, numéro thématique coordonné par Rousselet K. sur le thème : Pèlerinages en Eurasie et au-delà, vol.36, p.233-256.

Seraïdari K., 2019, « La valeur économique des lieux de culte grecs. Du pèlerinage au tourisme alternatif ", Archives de Sciences Sociales des Religions, numéro thématique coordonné par Makrides V. N. et Seraïdari K. sur le thème : Christianisme orthodoxe et économie dans le sud-est européen contemporain, n.185, p.87-106.

Skopetea E., 1988, Le Royaume modèle » et la Grande Idée. Vues sur le problème national en Grèce (1830-1880), Athènes, Polytypo, 456p. (en grec).

Stelaku V., 2008, « Space, place and identity. Memory and religion in two Cappadocian Greek settlements ", dans Hirschon R. (dir), Crossing the Aegean. An appraisal of the 1923 compulsory population exchange between Greece and Turkey, New York/Oxford, Berghahn Books, p.179-192.

Tantalidis I. D., 1874, Sur la question du monastère de saint Panteleimon du Mont Athos, Constantinople, Typois Vyzantidos, 209p. (en grec)

Vernezos I. D., 2009, « Saint Jean le Russe », p.1-8 (en grec), http://www.ecclesia.gr/greek/ holysynod/commitees/tourism/a5.pdf, date de consultation : mardi 12 novembre 2019.

\section{NOTES}

1. Dans le cadre du programme interdisciplinaire RICONTRANS (H2020-ERC-COG-2018), sous la direction de Juliana Boycheva et ayant comme thème : «Culture visuelle, piété et propagande : transfert et réception de l'art religieux russe dans les Balkans et la Méditerranée orientale ».

2. https://oir.gr/xenonas/domatia/, date de consultation : 25 novembre 2019 (en grec).

3. "L'évêque Chrysostomos, dans son livre, ainsi que le site Internet du pèlerinage (https:// oir.gr/o-ieros-naos/ieres-eikones/) donnent souvent la traduction des karamanlidika en grec.

4. Toutefois, dans une autre note du même livre, référence est faite au «problème aigu entre les moines grecs et russes qui voulaient élire leur propre higoumène »: à cause de cette crise, l'iconographe grec Daniil Katounakiotis (1846-1929), qui peignit beaucoup d'icônes pour l'église de saint Jean en Cappadoce, fut exilé du monastère de Panteleimon (ibid, p.178, note 258). 


\section{RÉSUMÉS}

L'église qui fut construite dans un village d'Eubée en 1951 en l'honneur de saint Jean le Russe, constitue un des lieux de pèlerinage les plus importants de Grèce. L'article examine le paramètre religieux, mémoriel et commercial de ce pèlerinage, ainsi que la dimension de territorialité qui se déploie à trois niveaux: a) les modèles d'identification des réfugiés qui apportèrent en 1924, après l'échange de populations entre la Grèce et la Turquie, la relique du saint de leur Cappadoce natale en Eubée ; b) le sentiment de familiarité des pèlerins russes qui viennent massivement rendre hommage à ce saint; et c) la logique d'appropriation. En adoptant une perspective diachronique, l'article montre le rôle fondamental que les Russes ont joué dans l'établissement de ce culte dès 1881 - date à la quelle l'avant-bras droit de la relique du saint fut offert aux moines russes du monastère Panteleimon du Mont Athos. Des offrandes russes du dix-neuvième siècle, exposées dans le musée qui se trouve près du sanctuaire, attestent de cette continuité et inscrivent les visiteurs russes actuels dans une lignée de pèlerins. Dans ce cas, les marqueurs de territorialité servent à tracer les limites entre familiarité et appropriation : le saint était, certes, leur compatriote, mais il a toujours voulu rester avec les orthodoxes de Prokopi, les premiers à reconnaître sa sainteté avant même son trépas. Alors que les offrandes russes du dix-neuvième siècle donnent de la profondeur temporelle à la relation que les pèlerins russes entretiennent avec le saint, pour les descendants des réfugiés, la dimension de territorialité qui leur est associée prend une autre forme: elles sont une source de fierté (en tant que preuve de la réputation internationale de leur saint) mais aussi un héritage légué par leurs ancêtres cappadociens, les Karamanlides. Cette population orthodoxe parlait le turc et l'écrivait avec des caractères grecs : comme leurs descendants aiment le répéter, les orthodoxes de Prokopi ont préféré perdre l'usage de la langue grecque plutôt qu'abdiquer la foi orthodoxe. Alors que la turcophonie continue d'être un élément perturbateur dans la construction de la rhétorique nationale grecque, la référence aux Russes parvient à "dés-ottomaniser " les orthodoxes turcophones de Prokopi, dans la mesure où les contacts réguliers entre les deux groupes commencèrent après une période d'affrontements militaires entre l'Empire russe et l'Empire ottoman, avec la guerre de Crimée d'abord, puis avec la guerre russo-turque de 1877-1878. Les offrandes russes du dix-neuvième siècle, mais aussi «le coin russe » à l'intérieur de l'église actuelle qui se trouve à côté du reliquaire et qui est décoré avec des fresques de saints russes, attestent de la capacité de ce lieu de pèlerinage à renvoyer à d'autres espaces (comme la Cappadoce, le Mont Athos ou la Russie) et à décliner, sous différentes formes, la dimension de territorialité.

Constructed in a locality of Evia in 1951, the church of Saint John the Russian constitutes one of the most important pilgrimages of Greece. The article examines the religious, memorial and commercial parameters of this pilgrimage, as well as the dimension of territoriality and its deployment on three levels: a) the models of identification of the refugees who brought the relic of the saint from their native Cappadocia to Evia in 1924, after the population exchange between Greece and Turkey; b) the feeling of familiarity of Russian pilgrims who come massively to pray in front of the relic; and c) the logic of appropriation. Adopting a diachronic perspective, the article shows the fundamental role Russians have played in the establishment of the saint's worship since 1881 - when the forearm of the saint's relic was offered to Russian monks at the monastery of Panteleimon of Mount Athos. The Russian offerings of the nineteenth century, which are exposed in the museum next to the shrine, bring the proof of this continuity and inscribe current Russian visitors into a "lineage" of pilgrims. Through these marks of territoriality, the limits between familiarity and appropriation are redefined: although the saint 
was their compatriot, he has always decided to stay with those who were the first to recognize his sanctity (even before his death), that is the Orthodox of Prokopi. If the Russian offerings of the nineteenth century give temporal depth to the relationship between the saint and the Russian pilgrims, the dimension of territoriality that these objects evoke, takes another form in the case of the refugees' descendants: for them, the Russian offerings are a source of pride (proving the international reputation of theirsaint) but also the legacy of their Cappadocian ancestors, the Karamanlides. This Orthodox population was speaking Ottoman Turkish, but wrote in Greek characters: as their current descendants stress, the Orthodox of Prokopi decided to lose the use of Greek language in order to be able to keep their Orthodox faith. Although this Turkishspeaking population constitutes a challenge to the Greek national rhetoric, the reference to the Russians manages to "des-Ottomanize" the Orthodox of Prokopi, since the regular contacts between the two groups started at the end of a period of military confrontations between the Russian and the Ottoman Empire (the Crimean War and the Russo-Turkish War of 1877-1878). The Russian offerings of the nineteenth century, but also the "Russian corner" (decorated with frescos of Russian saints) that one's can find next to the reliquary of the saint, show the capacity of this site of pilgrimage to evoke other spaces (like Cappadocia, Mount Athos or Russia) and to develop, in a variety of forms, the dimension of territoriality.

INDEX

Mots-clés : Pèlerinage, réfugiés, relique, territorialité, Empire Ottoman, Cappadoce, Grèce Keywords : Pilgrimage, refugees, relic, territoriality, Ottoman Empire, Cappadocia, Greece

\section{AUTEUR}

\section{KATERINA SERAÏDARI}

Chercheuse associée au Centre d'Anthropologie sociale-LISST à Toulouse et à l'Institute for Mediterranean Studies-IMS, Grèce/programme RICONTRANS 